\title{
Effect of mesostructure on strain rate dependent behaviour in high strength steel sheets
}

\author{
K. Natori ${ }^{1}$, R. Kishi ${ }^{1}$, H. Shimahara ${ }^{2}$, Y. Arao ${ }^{3}$ \& T. Tanaka ${ }^{3}$ \\ ${ }^{1}$ Graduate School of Engineering, Doshisha University, Japan \\ ${ }^{2}$ Hilti Corporation, Liechtenstein \\ ${ }^{3}$ Faculty of Science and Engineering, Doshisha University, Japan
}

\begin{abstract}
The mechanism of strain rate dependence on the Bauschinger effect in dualphase type high strength steel sheets is investigated and the influence of mesostructure differences on spring-back is estimated in this study. Compression-tensile tests at a strain rate from $0.007 / \mathrm{s}$ to $100 / \mathrm{s}$ is conducted. To differentiate between the multiphase structure-induced effect and the matrix properties, the same tests are also conducted for ferrite single phase steel. As a result, the Bauschinger effect on dual phase steel is not associated with the matrix properties independently. Moreover, it is revealed that a multiphase structure-induced effect acts a dominant function for deformation characteristics through experiments and micro observations.

Keywords: sheet metal forming, Bauschinger effect, strain rate dependence, high strength steel, dual phase, multiscale analysis, mesoscale structure.
\end{abstract}

\section{Introduction}

An improvement in automobile fuel consumption toward the realization of a low carbon society and earth environment protection is greatly needed. A high strength steel sheet (HSS), especially the dual-phase type, is adopted as a composition material of an automobile body and contributes to both weight saving and improvement in safety. However, although the practical use in automobiles is being promoted by the development of material technology and forming technology, HSS is still considered a difficult-to-form material. The serious problem of HSS in press forming is that a spring-back often occurs and 
with significant complications. As a solution to this problem, the forming die is designed based on the predicted spring-back angle by numerical simulation. To improve simulation accuracy, a material model considering the Bauschinger effect based on a quasi-static experiment was developed [1]. Furthermore, an attempt to reduce the spring-back angle by controlling punch speed and motion has been actively studied (see, for example, [2]). Therefore, we note that the considerable problem is the influence of strain rate.

Additionally, while it is an established theory with the mechanism of the Bauschinger effect that back stress is generated because dislocation is piled up at obstacles during pre-deformation, it has been pointed that local stress distribution resulting from the presence of a secondary hard phase becomes a factor of the Bauschinger effect [3]. Actually, the Bauschinger effect in dual-phase metal should be subjected to influence by the combination of the matrix properties and the multiphase structure-induced effect. In recent years, to give an equal emphasis on strength and formability of metal materials, a strengthening mechanism with controlling the mesoscale structure is actively developed rather than using the existing microscale mechanism, e.g. precipitation or solid solution. For this reason, it is required to recognize the multiphase structure-induced deformation mechanism. Furthermore, a material with such multiphase structure is interpreted to have severely strain rate dependence on deformation characteristics while being superior to impact absorption capability [4].

Then, the purpose of this study is to investigate the mechanism of strain rate dependence on the Bauschinger effect in dual phase steel and to estimate the influence of mesostructure differences on spring-back. We validate a hypothesis derived from an experimental evaluation test for the Bauschinger effect with simulation using a simplified analytical model having two phase structures.

\section{Bauschinger effect}

When stress is applied to metal materials in a different direction to predeformation, yield stress and flow stress decrease significantly compared with the case that the stress direction is equal to pre-deformation. These phenomena have been called the Bauschinger effect. In the case of single phase metal, the back stress arises as a resistance to long range obstacles, for example, piled up dislocation in the cell-wall and grain boundary or sessile dislocation; the Bauschinger effect occurs by the presence of the back stress which helps reversal deformation at the next different stress path. And, with regard to metal materials containing a hard secondary phase, it has been recognized that the Bauschinger effect occurs due to an early yield phenomenon attributed to local strain gradient resulting from a strength differential between the primary and secondary phases [3].

A deformation resistance force can be considered to be two separate components which are independent and dependent of the direction of the stress path. The former is a resistance from forest dislocation and yield stress determined by the metal structure, and the latter is a back stress. The mean back stress is derived from the following equation [5]. 


$$
\text { backstress }=\frac{1}{2}\left(\left|\sigma_{\text {reverse }}\right|-\left|\sigma_{\text {reyield } \Delta \varepsilon}\right|\right)
$$

In this study, in order to represent a scale of the Bauschinger effect directly, the ratio of Bauschinger effect, B.E.R., is calculated from the following equation [6]. It indicates a decreasing rate of yield point, and is an evaluation index in which back stress is made dimensionless by the mechanical property.

$$
\text { B.E.R. }=\frac{\left|\sigma_{\text {reverse }}\right|-\left|\sigma_{\text {reyield } \Delta \varepsilon}\right|}{\left|\sigma_{\text {reverse }}\right|}
$$

Figure 1 depicts the details of parameters which are used in eqns. (1) and (2). B.E.R. as defined eqn. (2) shows the scale of Bauschinger effect. If B.E.R. is equal to zero, the Bauschinger effect does not occur; the Bauschinger effect significantly occurs as the value of B.E.R. grows.

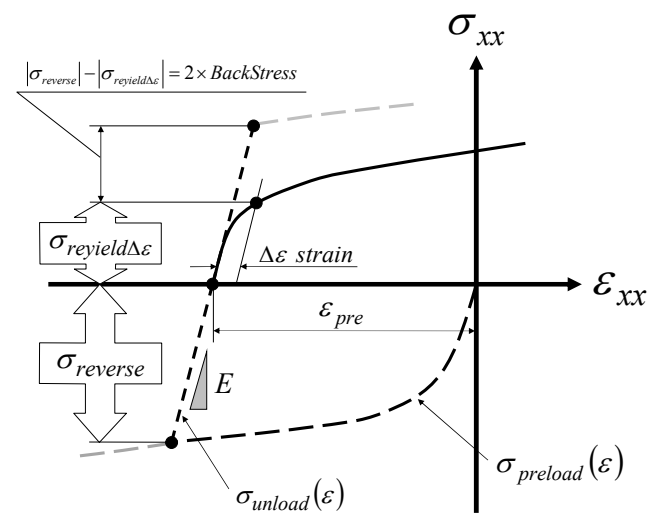

Figure 1: Description of the Bauschinger effect. $\sigma_{\text {reyield } \Delta \varepsilon}$ is yield stress in the re-load process as yield is evaluated at the point at which residual plastic strain $\Delta \varepsilon$ is indicated. $\sigma_{\text {reverse }}$ is the intersection point of the uniaxial history $\sigma_{\text {preload }}(\varepsilon)$ with the elastic unloading of compression force history $\sigma_{\text {unload }}(\varepsilon)$ which is calculated as pre-strain $\varepsilon_{\text {pre }}$ and Young's modulus $E$.

\section{Experimental details}

\subsection{Test materials}

The test materials are cold rolling high strength steel of a dual-phase type composed of ferrite and martensite, JSC590Y, and mild steel with a ferrite single phase, SPC270E (microstructure photographs are shown in fig. 2). We prepare 
two sheets of dual phase steel with a different production lot, JSC590Y-1 and -2. The mechanical properties of steels are listed in table 1 .

\subsection{Evaluation test for the Bauschinger effect}

In order to observe the level of Bauschinger effect [7, 8], we conduct a compression-tensile test with various strain rates and amounts of precompression; the stress-strain relationship in the tensile process is obtained. The decreasing rate of re-yield stress, B.E.R., and back stress corresponding to a shift of yield surface centre for the combined hardening rule are measured as an evaluation index for the Bauschinger effect using eqns. (1) and (2). The result of the uniaxial tensile test is substituted for $\sigma_{\text {preload }}(\varepsilon)$ in Fig. 1. The evaluation point corresponds $0.2 \%$ proof stress as yield stress $(\Delta \varepsilon=0.002)$. Additionally, we perform electron backscatter diffraction analysis (TSL Solutions K.K.) on the test-piece after compression by a scanning electron microscope (JEOL Ltd., JSM6301F) for calculation of kernel average misorientation (KAM).

(a)

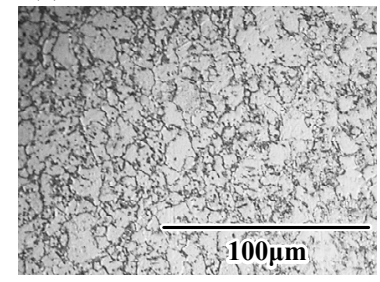

(b)

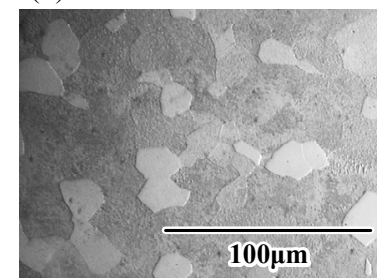

Figure 2: Microstructure observation. Etching: Nital 1.5\%. Microstructure of (a) HSS in DP type, JSC590Y-2, and (b) mild steel with ferrite single phase, SPC270E based on the Japan Iron and Steel Federation Standard. Whitish area; ferrite, Dark area; martensite.

Table 1: Mechanical property of test material steels along the rolling direction. Yield stress is identified as $0.2 \%$ proof stress; $n$ value is calculated as a point of $2 \%$ and $5 \%$ total strain. These data are obtained from the results of the quasi-static tensile test.

\begin{tabular}{lccc}
\hline Test mat. & $\begin{array}{c}\text { Yield stress } \\
(\mathrm{MPa})\end{array}$ & $\begin{array}{c}\text { Tensile strength } \\
(\mathrm{MPa})\end{array}$ & $\begin{array}{c}\mathrm{n} \text { value } \\
(2,5 \% \text { total strain })\end{array}$ \\
\hline \hline JSC590Y-1 & 395 & 615 & 0.171 \\
\hline JSC590Y-2 & 360 & 609 & 0.193 \\
\hline SPC270E & 128 & 285 & 0.290 \\
\hline
\end{tabular}

The test conditions are as follows: a dumbbell specimen, $12 \mathrm{~mm}$ in initial gauge length, $4.3 \mathrm{~mm}$ in width and $1.4 \mathrm{~mm}$ in thickness is prepared by cutting along the rolling direction using a wire-electric discharge machine [9]. 


\subsubsection{Compression test}

A quasi-static, 0.007/s, and dynamic, 10/s, 100/s, compression test is conducted; The universal testing machine (Shimadzu Corp., AG-100kNI) is used for the quasi-static test, and an electro hydraulic high speed compression testing machine (Shimadzu Corp., HTM-10kNI) is used for the dynamic test. A dedicated compression test jig, which has been developed in this study, is used to avoid buckling of the specimen.

\subsubsection{Tensile test}

A quasi-static, 0.007/s, and dynamic, 10/s, 100/s, tensile test [9] is conducted after compression; the universal testing machine (Shimadzu Corp., AG-100kNI) is used for the quasi-static test, and an electro hydraulic high speed tensile testing machine (Shimadzu Corp., EHF-U2H-20L) is used for the dynamic test. The dynamic test machine's actuator has been controlled by an electro hydraulic high speed servo valve during the approach process. Therefore, a deformation to the specimen has been caused at an expected speed. To reduce noise effects resulting from inertial force by the entire machine system, the load history at high speed deformation is measured by strain gauge adapting both sides of the grip part of the specimen. The strain history can be measured by a strain gauge adapting gauge part up to about 5\%. The exceeding strain is calculated from the mean strain rate which is measured by analysing the motion picture recorded by a high speed camera.

Table 2: Input parameters for the RVE numerical test. With regard to the ferrite model-I, -II and martensite, the experimental result under $0.007 / \mathrm{s}, 100 / \mathrm{s}$ (fig. 4(b)) and literature data [10] is referred to, respectively.

\begin{tabular}{l|c|c|c|}
\hline \multirow{2}{*}{ Parts } & \multicolumn{2}{|c|}{ Matrix } & Inclusion \\
Subject to modelling & I & Martensite \\
\hline \hline Hardening rule & \multicolumn{2}{|c|}{ Isotropic } & Isotropic \\
\hline $\begin{array}{l}\text { Consideration for strain rate } \\
\text { dependence of flow stress }\end{array}$ & \multicolumn{2}{|c|}{$(\checkmark)$} & - \\
\hline Elastic-plastic behaviour & \multicolumn{2}{|c|}{ Bilinear law } & Bilinear law \\
\hline $\mathrm{E}(\mathrm{GPa})$ & 195 & 195 & 210 \\
\hline $\mathrm{N}$ & 0.3 & 0.3 & 0.3 \\
\hline $\mathrm{YS}(\mathrm{MPa})$ & 125.4 & 385.6 & 1339.8 \\
\hline $\mathrm{H}(\mathrm{MPa})$ & 1375.6 & 2162.3 & 4688.0 \\
\hline
\end{tabular}

\section{Numerical analysis}

\subsection{Numerical material test for RVE}

A three dimensional simplified representative volume element (RVE), composed of a soft and hard phase is deformed in simulation. RVE is created by the multiscale structure modelling platform, Digimat (e-xstream engineering). Ferrite is 
modelled as a matrix and martensite is modelled as spherical inclusions which are $0.08-0.3$ in diameter and 0.30 volume fractions of RVE $(1 \times 1 \times 1)$. Numerical analysis is performed using the nonlinear dynamic explicit finite element code, LS-DYNA. Ideal elastic-plastic which is simulated ferrite and martensite is applied as mechanical properties listed in table 2. While the strain rate dependence of the flow stress is not considered in both phases, two kinds of behaviour in the ferrite phase are set with reference to the experimental result of the uniaxial tensile test in a quasi-static and dynamic state. The FEM model is meshed by a four-node tetra constant strain element; it has 9678 nodes and 49,791 elements.

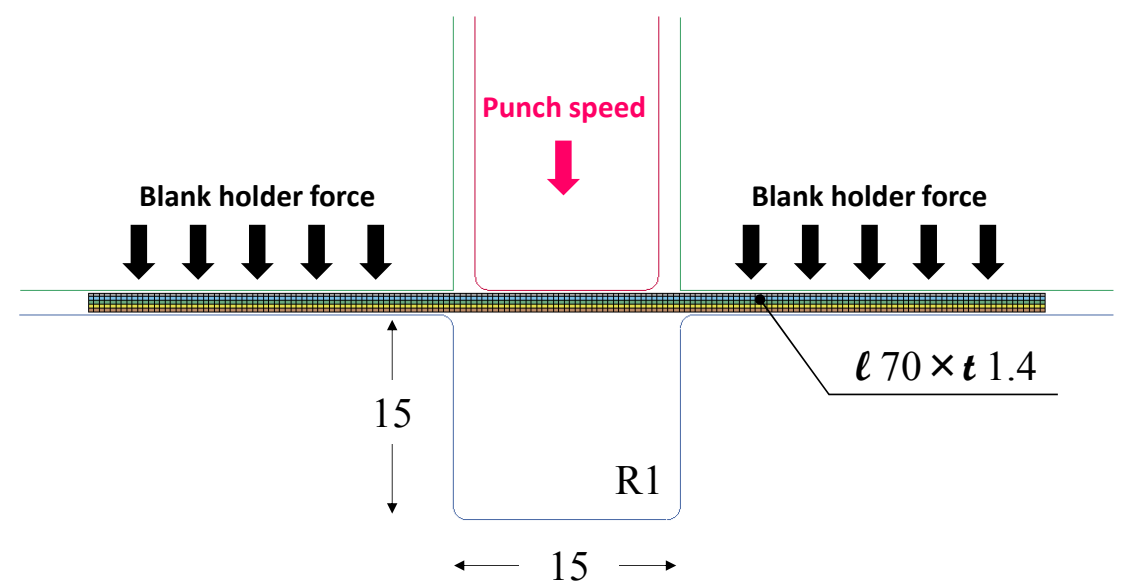

Figure 3: Schematic analysis condition for hat-shaped forming with a punch speed of $10-1000 \mathrm{~mm} / \mathrm{s}$. It is assumed that the jig has a rigid body and 0.1 friction coefficient with the workpiece, and the blank holder force is kept at $30 \mathrm{kN}$. Unit: $\mathrm{mm}$.

Table 3: Input parameters for hat-shaped forming analysis. DP steel sheets are modelled based on the experimental result (figs 4(a) and 5 (a)).

\begin{tabular}{|l|c|c|}
\hline Part & \multicolumn{2}{|c|}{ Workpiece } \\
\hline \hline Hubject to modelling & JSC590Y-1 & JSC590Y-2 \\
\hline $\begin{array}{l}\text { Consideration for strain rate } \\
\text { dependence of flow stress }\end{array}$ & Combined & Combined \\
\hline Elastic-plastic behaviour & Bilinear law & Bilinear law \\
\hline $\mathrm{E}(\mathrm{GPa})$ & 193 & 193 \\
\hline$v$ & 0.3 & 0.3 \\
\hline YS $(\mathrm{MPa})$ & 307.7 & 314.6 \\
\hline H' $(\mathrm{MPa})$ & 2980.0 & 2911.3 \\
\hline Cowper-Symonds eq. & $\mathrm{C}=4798.1, \mathrm{P}=9.191$ & $\mathrm{C}=920.7, \mathrm{P}=8.757$ \\
\hline $\begin{array}{l}\text { Parameter for the } \\
\text { hardening rule }\end{array}$ & $\begin{array}{l}\text { Input a coefficient according to } \varepsilon_{\text {pre }} \text { of each } \\
\text { layer which is calculated from exploratory } \\
\text { analysis. }\end{array}$ \\
\hline
\end{tabular}




\subsection{Numerical hat shaped forming}

Sheet forming analysis is performed using LS-DYNA by introducing the experimental data of the Bauschinger effect resulting from the compressiontensile test as a combined hardening model, then the spring-back is analysed by an implicit method (a brief configuration is shown in fig. 3). Input mechanical properties data for the workpiece are listed in table 3 . The sheet metal is modelled to separate five layers by a four-node plane strain shell element with full integration; it has 1056 nodes and 874 elements.

\section{Results and discussion}

\subsection{Comparison of dual phase steel with matrix single phase steel}

\subsubsection{Strain rate dependence on the Bauschinger effect}

Figure 4 shows the tensile test result for DP steel and ferrite single phase steel with pre-compression under various conditions. It is apparent that the strain rate is dependent on the flow stress. Mild steel depends strongly on the strain rate
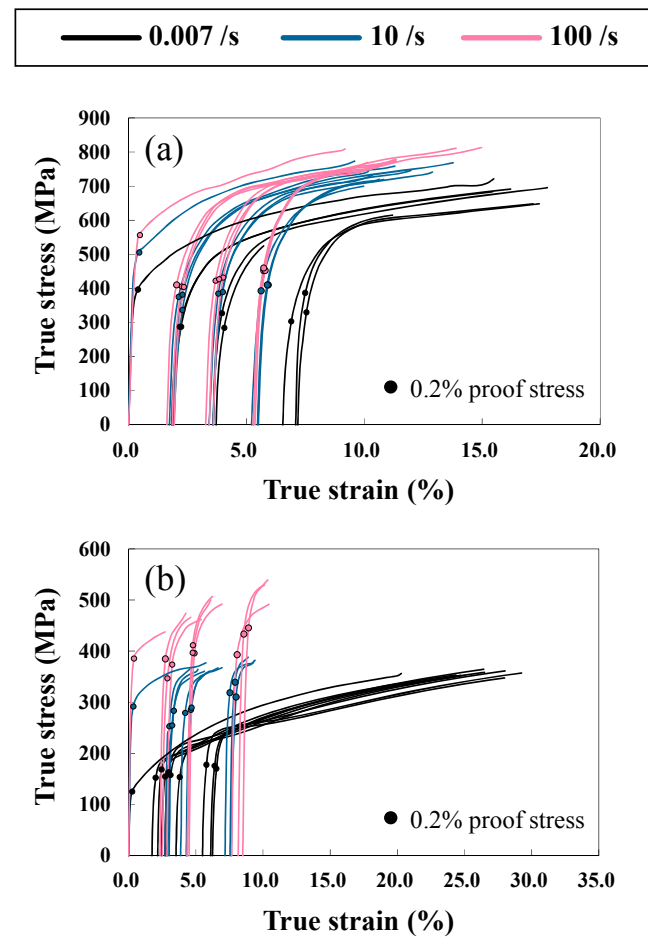

Figure 4: The stress-strain response of (a) JSC590Y-1 and (b) SPC270E after application of various pre-deformations. The pre-compression strain is indicated as a shift-amount from the origin of the horizontal axis. 
because of having strengthening mechanisms which are subjected to thermal activation. Next, an evaluation index for the Bauschinger effect is calculated from curves of fig. 4 and is shown in fig. 5. It indicates a strain rate dependence on the Bauschinger effect; that is, the decreasing rate of re-yield stress is lowering with an increasing strain rate. Ferrite single phase steel can be assumed to be an element of DP steel's matrix. However, there is a clear divergence in the dependence on the pre-strain amount. In fact, it is obvious from the experiment shown in fig. 5 that the Bauschinger effect in dual-phase metal should be subjected to influence by the multiphase structure-induced effect rather than the matrix properties.

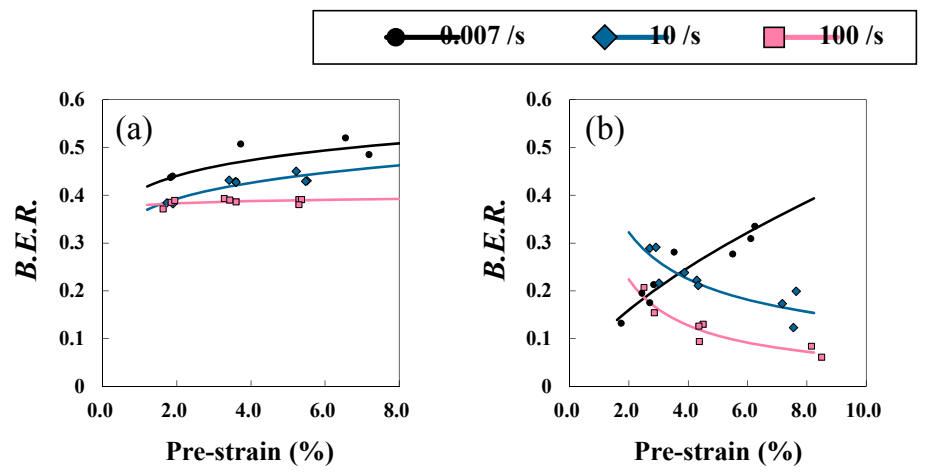

Figure 5: Decreasing rate of re-yield stress by the Bauschinger effect derived from eqn. (2). The dependence on the Bauschinger effect on the pre-strain amount differs between (a) JSC590Y-1 and (b) SPC270E under high speed deformation.

\subsubsection{Mechanism}

Figure 6 depicts crystal misorientation and phase mapping of DP steel after precompression. Crystal misorientation generated in a grain causes the introduction of geometrically necessary dislocation; this can be defined as local strain distribution in a broad sense. As shown in fig. 6(a), comparing part X with part $\mathrm{Y}$, with ferrite intertwined with martensite more deformation is caused. This contributes to the macroscopic Bauschinger effect and conforms with the report from computer simulation [3] as described in section 2 .

Figure 7 depicts the analysis result regarding RVE composed of ferrite and martensite with and almost without having the strain rate dependence of flow stress, respectively. The gap of back stress continues to widen with increasing pre-strain; and back stress is enlarged (as shown in fig. 7(a)) and local strain in the matrix shows a wide distribution with the same macroscopic pre-strain (as shown in fig. 7(b)) when the deformation strain rate is low; that is, the difference between the matrix and inclusion strength is extended. This means that a part which is easy to be subjected to deformation continues to deform. Then, reversing the stress direction, a part with low local pre-strain is yielded earlier and great back stress is generated. Therefore, the strain rate dependence of flow 
stress on the matrix material is one of the factors why the strain rate dependence of the macroscopic Bauschinger effect occurs.

(a)

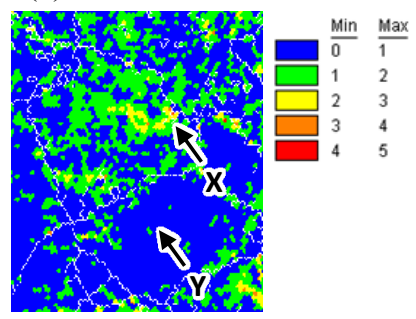

(b)

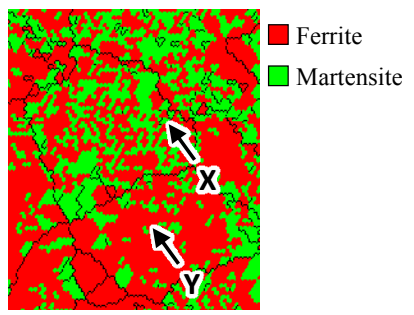

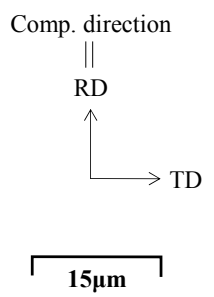

Figure 6: Observation of the surface of JSC590Y-2 with $6.41 \%$ precompression measured by EBSD: (a) depicts the KAM mapping calculated from crystal orientation; (b) depicts the phase mapping identified bcc and bct lattice as ferrite and martensite, respectively.

(a)

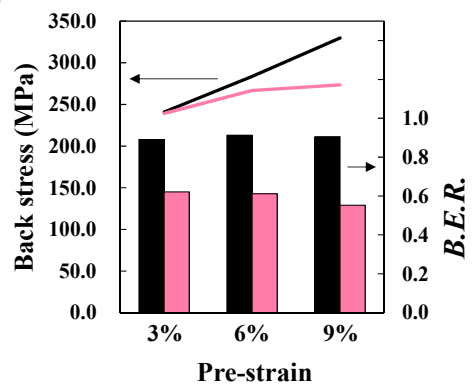

(c)

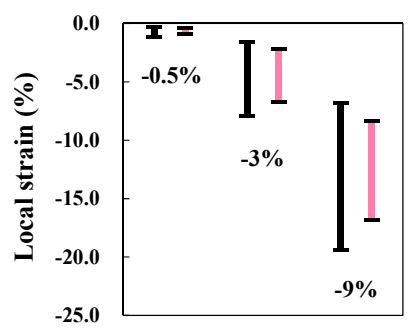

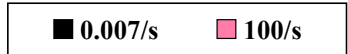

(b)

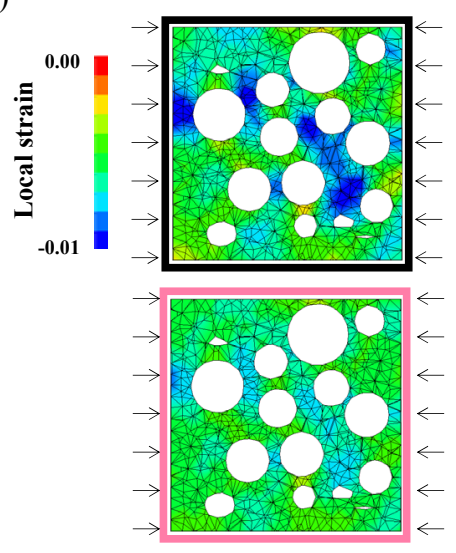

Figure 7: Analysis result of compression-tensile test for RVE composed of two-phase structures. The isotropic hardening rule is applied to the properties of both phases: (a) is the evaluation index for the Bauschinger effect derived from eqns. (1) and (2); (b) depicts contour figures of strain along the loading direction at a crosssection of RVE at macro pre-deformation of $-0.5 \%$; (c) shows the progress in distribution width of local strain on each macro prestrain. 


\subsection{Comparison between dual phase steel with a variation of production lot}

\subsubsection{Error of mechanical properties}

Figure 8 shows the deformation properties on two sheets of DP steel. Under high speed deformation, the sensitivity of the scale of Bauschinger effect to the variation of lot is increased. To investigate the gaps, we prepared a simplified RVE model, an inclusion of which has a bigger volume fraction $(6 \%)$, bigger size $(60 \%)$ and lower strength (10\%) than the model introduced in section 5.1.2. It was found that an insignificant difference of the dual-phase structure was easy to trigger the gaps in the scale of the Bauschinger effect (in this case, over $5 \%$ of B.E.R. was changed) while the stress-strain relationship was not so different between the two models.

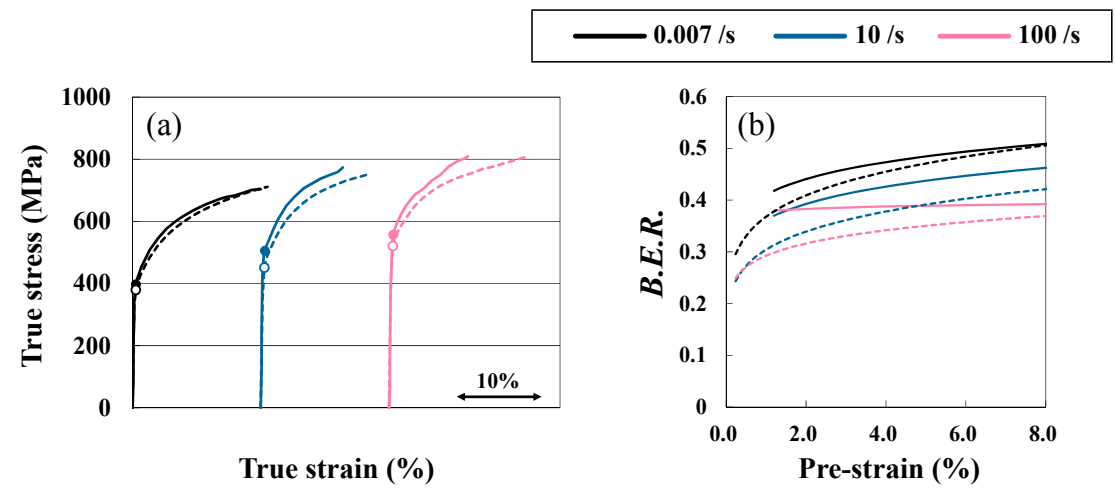

Figure 8: Influence of a difference in mesostructure configuration on deformation characteristics: (a) shows uniaxial tensile behaviours; (b) plots the evaluation index for the Bauschinger effect derived from eqn. (2). —— JSC590Y-1, - - - ; JSC590Y-2.

\subsubsection{Influence on spring-back prediction}

In order to investigate how much the gaps are involved in the spring-back angle, a hat shaped forming analysis is performed and the result reached are shown in fig. 9. In this situation, spring-back is less affected by production lot under high speed deformation. However, it is apparent that the prediction shows a low accuracy unless the strain rate dependence of the Bauschinger effect is considered by focusing on the difference between fig. 9 (b) and fig. 9(c). 


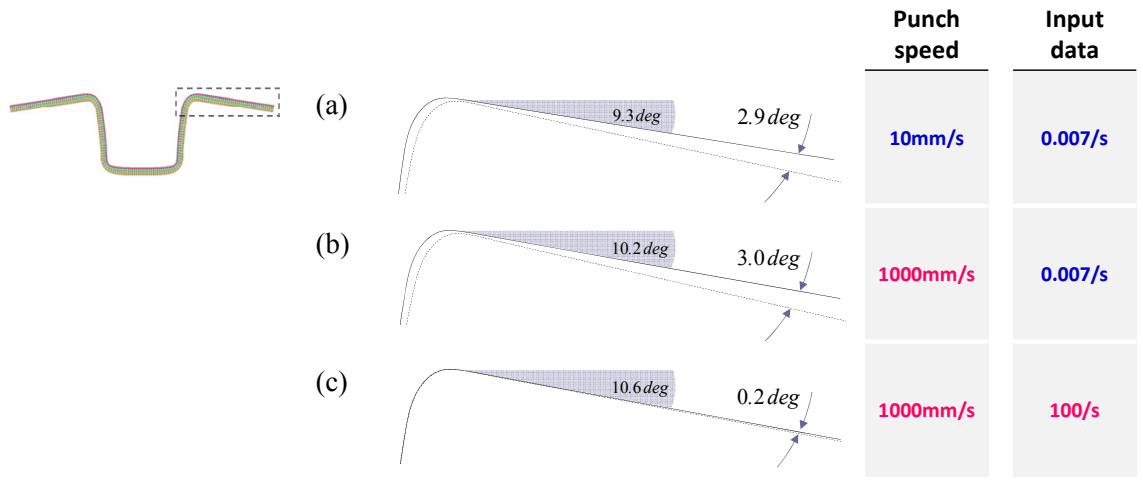

Figure 9: $\quad$ Spring-back analysis result. — ; JSC590Y-1, - - - ; JSC590Y-2. Forming is simulated under (a) quasi-static, (b) and (c) high speed. Parameters for the combined hardening rule are input with reference to each experimental data on the far right list.

\section{Conclusion}

To investigate the strain rate dependence on the Bauschinger effect in high strength dual-phase steel, we conducted a compression-tensile test at a strain rate from $0.007 / \mathrm{s}$ to $100 / \mathrm{s}$. To differentiate between the multiphase structure-induced effect and the matrix properties, the same tests were also conducted for ferrite single phase steel. The results we obtained are as follows:

1) The Bauschinger effect in dual phase steel is not associated with the matrix properties independently. Moreover, a multiphase structure-induced effect acts a dominant function for the deformation characteristics.

2) Early yield phenomenon occurs easily because the local strain in the matrix shows a wide distribution when increasing the difference between the matrix and the inclusion strength.

3) Under high speed deformation, the sensitivity of the scale of the Bauschinger effect to the variation of production lot in dual phase steel is increased. This affects the amount of spring-back.

\section{Acknowledgement}

This work was supported by JSPS Grant-in-Aid for JSPS Fellows No. 24-5201.

\section{References}

[1] Yoshida, F. \& Uemori, T., Int. J. Mech. Sci., 45(10), pp. 1687-1702, 2003.

[2] Sugamuma, S., J. Soc. Tech. Plasticity, 49(565), pp. 22-26, 2008.

[3] Matsui, K., Terada, K., Akiyama, M., Kuboki, T. \& Oikawa, K., Trans. Japan Soc. Mach. Eng. A, 68(675), pp. 71-78, 2002. 
162 High Performance and Optimum Design of Structures and Materials

[4] Urabe, T. \& Hosoya, Y., J. Soc. Tech. Plasticity, 46(534), pp. 16-20, 2005.

[5] Mori, Y., Ashida, U., Kishi, T. \& Horiuchi, R., Tetsu-to-Hagane, 64(9), pp. 1414-1423, 1978.

[6] Han, K., Vantyne, C. J. \& Levy, B. S., Metal. Mater. Trans. A, 36(5), pp. 2379-2384, 2005.

[7] Watanabe, K., Yoshihara, S., Natori, K., Tanaka, T. \& Imaida, Y., J. Soc. Tech. Plasticity, 51(594), pp. 48-53, 2010.

[8] Watanabe, K., Natori, K., Tanaka, T. \& Imaida, Y., WIT Trans. on the Built Environment, 112, pp. 119-131, 2010.

[9] Mukai, Y. \& Watanabe, K., Kobe Steel Eng. Report, 52(3), pp. 32-34, 2002.

[10] Nakamachi, E., Yokoyama, N., Fujita, K., Chen, Y., Morita, K. \& Morimoto, H., Trans. Japan Soc. Mach. Eng. A, 69(687), pp. 1595-1600, 2003. 